Proceedings

\title{
Flexible Supercapacitor Fabricated on a Polyester-Cotton Textile $^{+}$
}

\author{
Sheng Yong *, Stephen Beeby and Kai Yang
}

Citation: Yong, S.; Beeby, S.; Yang, K. Flexible Supercapacitor Fabricated on a Polyester-Cotton Textile. Proceedings 2021, 68, 7. https://doi. org/10.3390/proceedings2021068007

Published: 11 January 2021

Publisher's Note: MDPI stays neutral with regard to jurisdictional claims in published maps and institutional affiliations.

\section{Copyright: (C) 2021 by the authors.} Licensee MDPI, Basel, Switzerland. This article is an open access article distributed under the terms and conditions of the Creative Commons Attribution (CC BY) license (http://creativecommons.org/licenses/by/4.0/).
Smart Electronic Materials \& System Research Group, School of Electronics and Computer Science, University of Southampton, Southampton SO17 1BJ, UK; spb@ecs.soton.ac.uk (S.B.); ky2@ecs.soton.ac.uk (K.Y.)

* Correspondence: sy1v16@soton.ac.uk; Tel.: +44-238-052-3119

+ Presented at the International Conference on the Challenges, Opportunities, Innovations and Applications in Electronic Textiles (E-Textiles 2020), Virtual Venue, UK, 4 November 2020.

\begin{abstract}
This paper reports the design, fabrication and characterization of a flexible supercapacitor on top of a polyester-cotton textile. The textile-based, flexible supercapacitors were implemented with inexpensive screen-printed carbon black electrodes, an integrated polymer separator and a nonhazardous organic electrolyte. The encapsulated devices demonstrated area capacitance of 0.54 $\mathrm{mF} \cdot \mathrm{cm}^{-2}$.
\end{abstract}

Keywords: supercapacitor; e-textile; polymer membrane separator; flexible encapsulation

\section{Introduction}

Electronic textiles (also known as smart fabrics or e-textiles) are the integration of electronic functionality within woven, knitted and nonwoven textiles. At present, such systems are typically powered by conventional inflexible batteries which require frequent replacement [1] and/or need to be physically removed before washing. Therefore, a flexible energy storage device such as a supercapacitor integrated on top of or inside a textile are of great interest as the electrical energy supply for e-textile applications. Desirable characteristics include good cycling stability and high levels of energy and power density.

A supercapacitor is an electrochemical device for energy storage/buffering applications. It stores more electrical energy than a conventional capacitor while having better power density and cycling stability than a rechargeable battery. An electrical double-layer type supercapacitor is assembled with two electrode interfaces, each having opposite polarity with respect to the electrolyte solution. Textile-based supercapacitors can be used as an energy reservoir for e-textile systems. Previously, a textile supercapacitor was made with a pair of corrosive oxide material coated textile electrodes [2], discrete filter paper separator and acidic electrolyte [3]. These devices require extra care when packaging to minimize skin irritation. Therefore, although such supercapacitors may demonstrate good electrochemical performance, the use of hazardous materials and the requirement for multiple layers on textile mean they are not easy to integrate in practical e-textile applications.

Previously, Yong et al. [4] fabricated a supercapacitor in a single layer of woven cotton. The proposed device used cotton textile as a separator and spray-coated activated carbon as electrodes; the spray coating process was precisely controlled to not let the top and bottom electrodes touch each other and prevent device short circuits. The device was impregnated with a gel electrolyte and demonstrated area capacitance of $49.1 \mathrm{mF} \cdot \mathrm{cm}^{-2}$. Alternatively, Yong et al. [5] implemented a supercapacitor in a single layer of woven polyester-cotton textile. In this work, a copolymer membrane was fabricated in the polyester-cotton textile with screen printing and the phase invention process. The membrane acted as separator, physically separating the activated carbon electrodes spray coated on 
both sides of polyester-cotton textile. This device was impregnated with an organic electrolyte and encapsulated by an ethylene-vinyl acetate (EVA) polymer film applied using a heat press and achieved area capacitance of $13.1 \mathrm{mF} \cdot \mathrm{cm}^{-2}$. Manjakkal et al. [6] demonstrated a symmetrical supercapacitor on cellulose/polyester cloth. The electrodes were poly(3,4-ethylenedioxythiophene): poly(styrene sulfonate) and the cellulose/polyester cloth acted as the separator. This device demonstrated area capacitance of $10 \mathrm{mF} \cdot \mathrm{cm}^{-2}$. In these examples, the supercapacitors were fabricated by exploiting the structure of the textile itself, which provides a convenient mechanical scaffold for the electrodes. However, the consequence of this approach is that the supercapacitor electrodes are not on the same side of the textile. In e-textiles, the electronic functionality is typically realized on the one side of textile and therefore, powering such systems with the previously demonstrated single-layer supercapacitors will require an electrical connection to both sides of the textile, which will add further complexity to the assembly and further reduce the flexibility of the e-textile.

This paper presents an approach for implementing a flexible supercapacitor on top of the textile, enabling all functional layers to be integrated and encapsulated alongside the e-textile electronics. The electrodes of the supercapacitor were fabricated on top of a polyester-cotton textile using a simple screen printing process and inexpensive carbon black paste. The separator was implemented between the electrodes and the supercapacitor was encapsulated and tested with an organic electrolyte in order to study their electrochemical performance.

\section{Materials and Methods}

Figure 1 shows the schematic of the supercapacitor on textile, which was fabricated with two parts. Part 1 was a polyester-cotton textile (thickness of $150 \mu \mathrm{m}$ ) with a copolymer membrane, silver and carbon black electrode layers. Part 1 was fabricated by screen printing commercially available silver polymer paste (TC-C4001, Fabinks, Southampton, UK) directly onto the polyester-cotton textile which formed the supercapacitor's bottom current collector. Then, the bottom electrode was screen printed on top of the silver current collector. The electrode ink contained a carbon black powder with a particle size of $30 \mathrm{~nm}$ mixed with a solvent and $40 \mathrm{wt} \%$ of EVA polymer binder. A copolymer solution containing EVA and polymethyl methacrylate (PMMA) was screen printed on top of the carbon black electrode and treated with the phase inversion process using a suitable solvent. It formed a white porous membrane on top of the carbon electrode. Part 2 of the supercapacitor comprised the top electrode and current collector printed on a separate ethylene vinyl acetate (EVA) film with a thickness of $1 \mathrm{~mm}$. This served the dual purpose of forming the second half of the supercapacitor structure and the top encapsulating film that protected the device. Both part 1 and part 2 of the device are shown in Figure 1b. The membrane-coated carbon electrode on textile and the carbon electrode on the EVA film were impregnated with a nonhazardous and ionic conductive organic electrolyte. The EVA film was then placed face-down on the textile, with the top electrode aligned to the polymer membrane. This film was attached to the textile simultaneously with a backside EVA film using a heat press that resulted in the flexible supercapacitor (Figure 1c). 
(a)

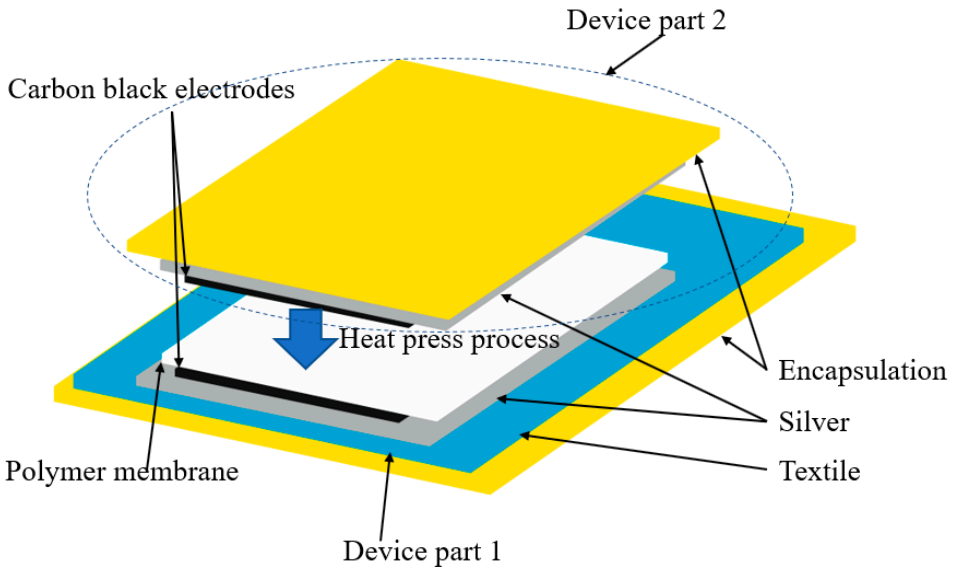

(b)

Device part 1
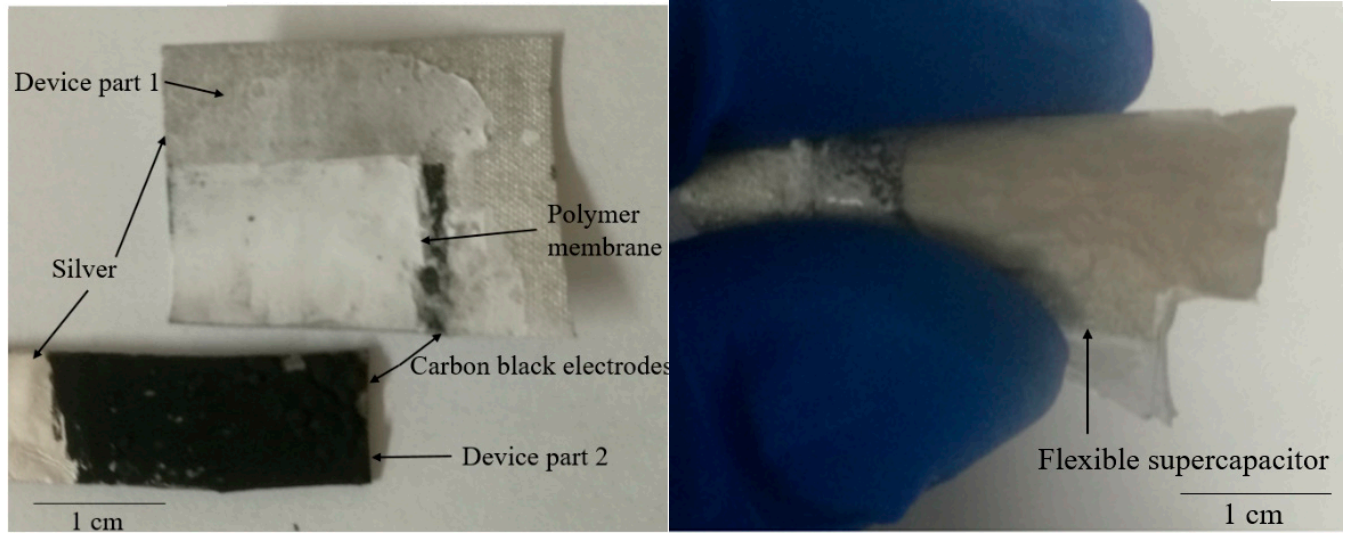

Figure 1. (a) Supercapacitor device schematic, (b) device part 1 and part 2, (c) flexible supercapacitor under 180 degrees of bending condition.

Figure 2 (left) shows the cross-sectional SEM picture of the part 1 textile electrode including the membrane, carbon and silver layer. The white part on the top of the picture is the membrane which is around $100 \mu \mathrm{m}$ thick. This copolymer membrane acted as the separator within the supercapacitor that prevented electrical short circuits but allowed ion transfer between the carbon black electrodes on the textile and EVA encapsulation films shown in Figure 2 (right). The thickness of the silver was $50 \mu \mathrm{m}$ and the thickness of screen-printed carbon layer was $200 \mu \mathrm{m}$.

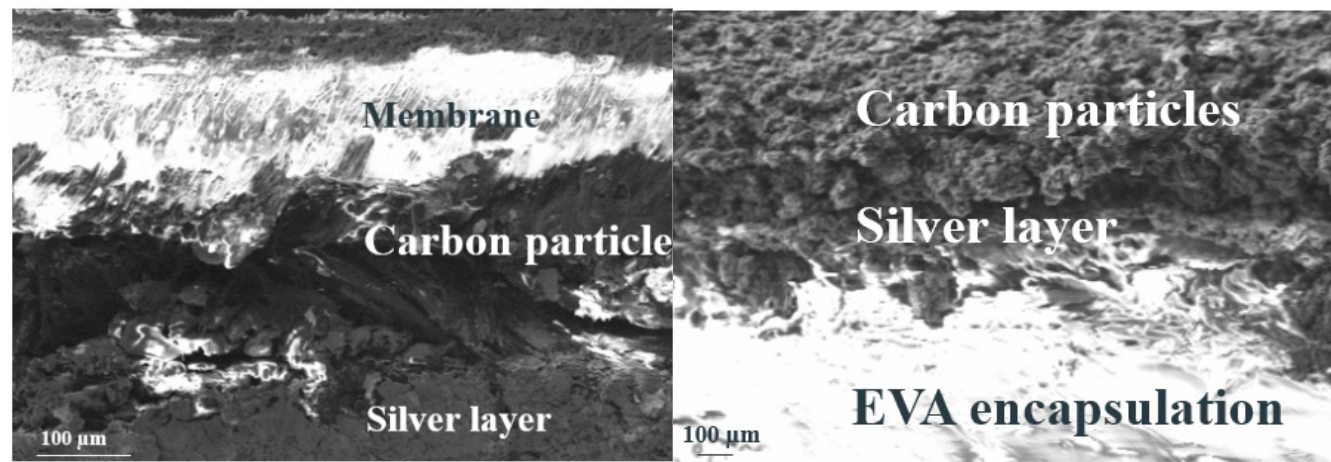

Figure 2. Cross-sectional SEM view of the (left) carbon black electrodes, silver layer on top of textile (with membrane), (right) EVA encapsulation film.

\section{Results}

The textile supercapacitor was tested using an Autolab pgsatat101 (Metrohm Autolab, Utrecht, The Netherlands). Each device had an active area of $0.8 \mathrm{~cm}^{2}$ with a total 
thickness of about $1.5 \mathrm{~mm}$ (including the thickness of EVA films that reduced during the heat press encapsulation process). The weight of the carbon materials in the active area was $4.2 \mathrm{mg}$ and accounted for $13.4 \%$ of the final device weight. The supercapacitor was characterized by cyclic voltammetry $(\mathrm{CV})$ at different scan rates and galvanostatic cycling (GC) at different scan voltages or currents.

The CV test results shown in Figure 3a indicate that the flexible supercapacitor on textile was electrochemically stable without the redox reaction at scan rates from 25 to $100 \mathrm{mV} \cdot \mathrm{s}^{-1}$. Its area capacitance varied between 0.70 and $0.42 \mathrm{mF} \cdot \mathrm{cm}^{-2}$ according to the scan rate. As shown in Figure $3 \mathrm{~b}$, the flexible supercapacitor on textile demonstrated area capacitance of $0.54 \mathrm{mF} \cdot \mathrm{cm}^{-2}$ with an equivalent series resistance (ESR) of $1.2 \mathrm{k} \Omega \cdot \mathrm{cm}^{-2}$ that depended on its test cycling current.

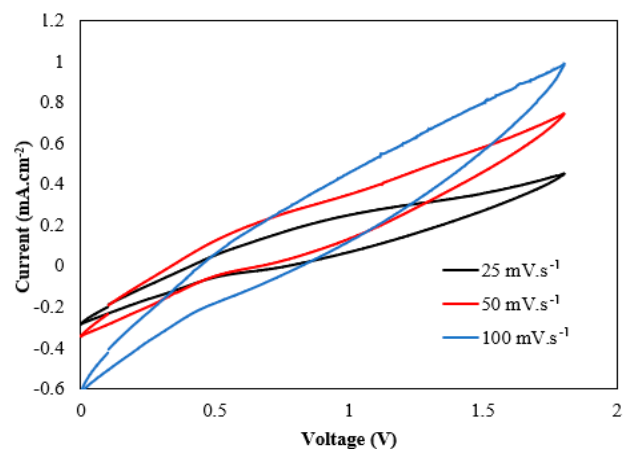

(a)

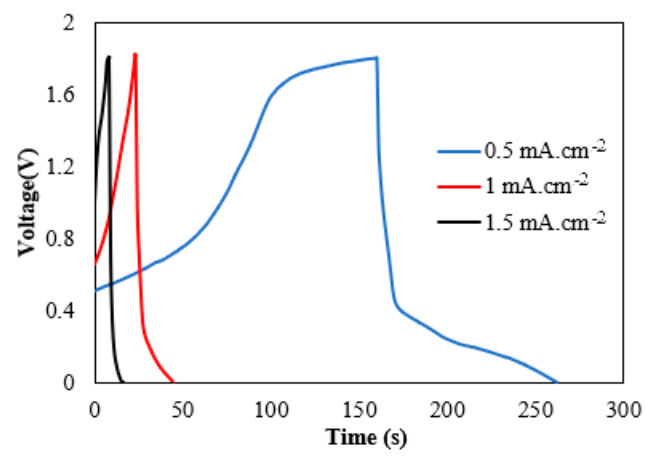

(b)

Figure 3. (a) CV tests between 0 and $1.8 \mathrm{~V}$ at the scan rates of 100,50 and $25 \mathrm{mV} \cdot \mathrm{s}^{-1}$. (b) GC tests between 0 and $1.8 \mathrm{~V}$ at the cycling currents of $0.5,1$ and $1.5 \mathrm{~mA} \cdot \mathrm{cm}^{-2}$.

Figure 4a shows that the flexible on-textile supercapacitor was electrochemically stable between -1.8 and $1.8 \mathrm{~V}$. This demonstrates the potential for the device to be used as an electrical power storage module in e-textile devices without suffering from short circuits. The encapsulated devices were repetitively bent around 90 degrees for 200 times, and the shape and value of the CV test results of the non-bent and bent devices demonstrated only a small difference. This shows that the flexible supercapacitor on textile was mechanically stable and could survive repeated bending. The flexible and durable copolymer membrane was stable under mechanical stress and the use of the screen-printing process with a flexible EVA polymer binder led to a uniform flexible and durable electrode layer.

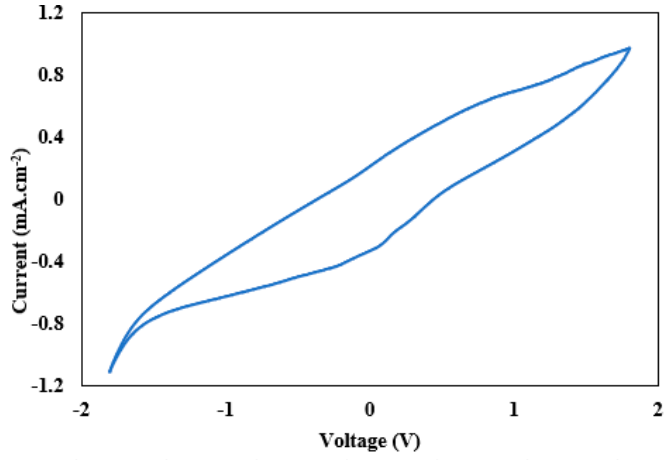

(a)

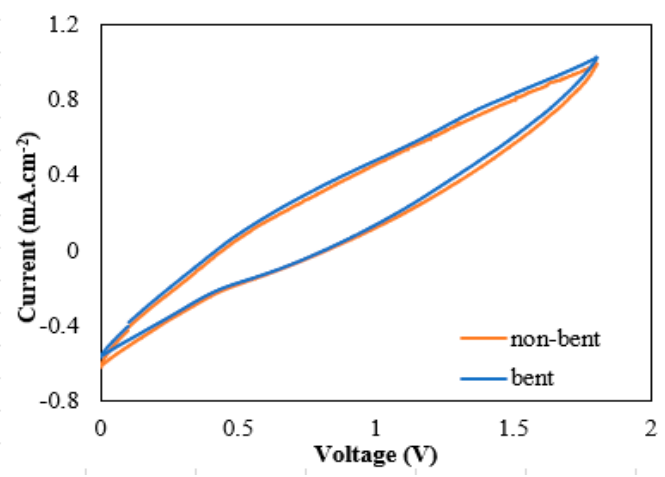

(b)

Figure 4. (a) CV test between -1.8 and $1.8 \mathrm{~V}$ at the scan rate of $100 \mathrm{mV} \cdot \mathrm{s}^{-1}$, (b) $\mathrm{CV}$ tests between 0 and $1.8 \mathrm{~V}$ at the scan rate of $100 \mathrm{mV} \cdot \mathrm{s}^{-1}$ for bent and non-bent devices at the scan rate of $100 \mathrm{mV} \cdot \mathrm{s}^{-1}$. 


\section{Discussion and Conclusions}

This paper reports a flexible and fully encapsulated supercapacitor fabricated on top of polyester-cotton which demonstrated area capacitance of $0.54 \mathrm{mF} \cdot \mathrm{cm}^{-2}$ and a normalized ESR of $1.2 \mathrm{k} \Omega . \mathrm{cm}^{-2}$. The proposed devices were electrochemically stable between $+/-1.8 \mathrm{~V}$ and exhibited negligible difference in $\mathrm{CV}$ tests between the bent and non-bent devices. In comparison with other works, these results are not as good as the encapsulated supercapacitor fabricated in the polyester-cotton textile with the copolymer membrane in the textile as a separator and activated carbon as electrodes [5], however, it serves to demonstrate the feasibility of the materials and fabrication processes and the assembly approach which has successfully realized an encapsulated supercapacitor on top of the polyester-cotton textile. Future work will include optimizing the formulation and fabrication process of the electrode and the electrolyte for better electrochemical performance.

Funding: This research was funded by EPSRC grants numbered EP/P010164/1 and EP/S001654/1.

Conflicts of Interest: The authors declare no conflict of interest.

\section{References}

1. Dias, T. Electronic Textiles Smart Fabrics and Wearable Technology; Woodhead Publishing: Sawston, UK, 2015.

2. Zhu, J.; Zhao, S.X.; Wu, X.; Wang, Y.F.; Yu, L.; Nan, C.W. Wrapping RGO/MoO2/carbon textile as supercapacitor electrode with enhanced flexibility and areal capacitance. Electrochim. Acta 2018, 282, 784-791.

3. Jin, H.; Peng, Z.H.; Tang, W.M.; Chan, H.L.W. Controllable functionalized carbon fabric for high-performance all-carbon-based supercapacitors. RSC Adv. 2014, 4, 33022-33028.

4. Yong, S.; Owen, J.; Beeby, S. Solid-state supercapacitor fabricated in a single woven textile layer for E-textiles applications. Adv. Eng. Mater. 2018, 20, 1700860.

5. Yong, S.; Hiller, N.; Yang, K.; Beeby, S. Integrated Flexible Textile Supercapacitor Fabricated in a Polyester-Cotton Fabric. Proceeding 2019, 32, 15.

6. Manjakkal, L.; Pullanchiyodan, A.; Yogeswaran, N.; Hosseini, E.; Dahiya, R. A Wearable Supercapacitor Based on Conductive PEDOT:PSS-Coated Cloth and a Sweat Electrolyte. Adv. Mater. 2020, 32, 1907254. 